УДК 537.22

\title{
Теоретические методы выявления нелинейных эффектов при термостимулированной деполяризации в твердых диэлектриках
}

\author{
B.А. Кальтка \\ Карагандинский государственный технический университет (Караганда, \\ Казахстан)

\section{Theoretical Methods of Nonlinear Effects Detection under Thermally Stimulated Depolarization in Solid Dielectrics}

V.A. Kalytka

Karaganda State Technical University (KSTU) (Karaganda, of Kazakhstan)

\begin{abstract}
Методами квазиклассической кинетической теории исследуется механизм протонно-релаксационной поляризации и термостимулированной деполяризации в кристаллах с водородными связями (КВС). Построены обобщенные выражения для комплексной диэлектрической проницаемости (КДП) и поляризации, выполняющиеся на основной частоте переменного поляризующего поля в бесконечном приближении теории возмущений по малому параметру. Установлено, что эффекты взаимодействия релаксационных мод объемной плотности заряда уже на основной частоте поля обусловливают аномально высокие поляризационные нелинейности, проявляющиеся в области сильных полей (10-1000 MB/м) и сверхвысоких температур (550-1500 К). Построены обобщенные нелинейные по полю выражения для коэффициентов кинетического уравнения, позволяющие выявить влияние параметров поля (амплитуда и частота ЭДС) на микроскопические акты перебросов физических релаксаторов (протонов) через потенциальный барьер. Из решения общего квазиклассического кинетического уравнения (для модели двойной симметричной потенциальной ямы с барьером параболической формы) построено выражение для плотности тока термостимулированной деполяризации в КВС, выполняющееся в широком диапазоне полей (100-1000 MB/м) и температур (1-1500 К). Предложена схема численного расчета характеристических параметров релаксаторов методом минимизации функции сравнения (МФС-методом).

Ключевые слова: кристаллы с водородными связями (КВС), комплексная диэлектрическая проницаемость

(КДП), статическая диэлектрическая проницаемость

(СДП), термостимулированный ток деполяризации.
\end{abstract}

This paper investigates the mechanisms of protonrelaxation polarization and thermally stimulated currents of depolarization (TCDP) in hydrogen bonded crystals (HBC) using methods of quasi-classical kinetic theory. Established generalized expressions for the complex dielectric permittivity (CDP) and polarization incorporates the main frequency of varying polarization field in the infinite approximation of perturbation theory with respect to a small parameter. It is revealed that that the effects of interaction among the relaxation modes of volume charge density starting from the main frequency of the field have already caused abnormally high polarizing nonlinearities in the range of strong fields $(10-1000 \mathrm{MV} / \mathrm{m})$ and ultra-high temperatures (550-1500 K). Generalized along the field expressions are established for kinetic equation coefficients. These expressions allow revealing reveal the influence of the field parameters (amplitude and frequency of the EMF) on the microscopic acts of transfer of physical relaxation oscillators (protons) through the potential barrier. The expression for the density of thermally stimulated currents of depolarization in HBC carried out in the wide range of fields (100-1000 MV/m) and temperatures $(1-1500 \mathrm{~K})$ is formulated on the basis of the general kinetic equation solution (for the model of a double symmetric potential well with a parabolic barrier). A numerical computation scheme for characteristic parameters oscillators is developed using the minimization comparison function method (MCF-method).

Key words: hydrogen bonded crystals (HBC), crystals with hydrogen bonds (CHB), complex dielectric permittivity (CDP), complex dielectric permeability (CDP), static dielectric permittivity (SDP), thermally stimulated currents of depolarization (TSDP).

DOI 10.14258/izvasu(2019)4-05 


\section{Введение}

Современный уровень развития техники и технологии требует дальнейшего усовершенствования существующих компьютерных методов анализа и прогнозирования свойств и параметров структуры твердотельных материалов, применяемых в качестве разнородных функциональных элементов (рабочих органов) в электротехнических схемах установок и систем (контрольно-измерительных, электронно-управляемых, диагностических и др.), работающих в условиях реального промышленного производства $[1,2]$.

Кристаллы с водородными связями (КВС), классифицируемые как протонные полупроводники и диэлектрики (ППД), находят широкое применение в электротехнической промышленности, технике высоких напряжений и изоляционной технике, электрохимических технологиях и водородной энергетике, электроэнергетике, лазерной технике, микроэлектронике и оптоэлектронике за счет характерных для материалов данного класса (МДП, МПМ-структуры) нелинейных электрофизических свойств [3-7]. Эти свойства проявляются в существенной зависимости электротехнических характеристик (диэлектрическая проницаемость, удельная объемная электрическая проводимость и др.) от параметров полей (амплитуда и частота ЭДС), температуры и от деформаций $[3,4]$.

Развитие аналитических схем исследования нелинейных электрофизических свойств КВС в широком диапазоне изменения параметров полей и температур является научно и технически важной задачей, определяющей направления и методы дальнейшего практического внедрения ППД в качестве электрооптических элементов, элементов электрически управляемых нелинейных конденсаторов, энергонезависимых запоминающих устройств на основе тонких пленок сегнетоэлектриков. Типичным представителем сегнетоэлектрических материалов с водородными связями является кристалл типа KDP, применяемый в лазерной технике (регуляторы параметров электромагнитного излучения и электрические затворы) [8-11]. Аналогичные KDP сегнетоэлектрические свойства проявляют и другие КВС (триглицин сульфат (ТГС) и сегнетова соль) [12-15].

1. Научная и практическая значимость результатов исследований. Постановка задачи исследования

При исследованиях электрофизических свойств КВС со сложной кристаллической структурой (слоистые силикаты (тальк, слюды, вермикулиты, глинистые минералы и др.), кристаллогидраты) наиболее эффективным является метод диэлектрической спектроскопии, сводящийся к измерению и расчету частотно-температурных спектров диэлектрических потерь $\operatorname{tg} \delta(\omega ; \mathrm{T})$ и температурных спектров токов термостимулированной поляризации (ТСТП) и деполяризации (ТСТД) $-J_{\text {depol }}(\mathrm{T})[1,2]$.
Теоретические представления о механизме термостимулированной деполяризации на настоящий момент времени базируются на феноменологической кинетической теории, сводящейся к решению системы уравнений Фоккера-Плака и Пуассона в линейном приближении теории возмущений [1] и позволяющей добиться хорошего совпадения с экспериментом при расчете параметров дефектов структуры в области достаточно высоких температур (150-350 К) [1,2]. Проблемная ситуация заключается в том, что в области низких температур (50-100 К) появляются заметные расхождения между расчетными и измеренными значениями энергии активации, а в области высокотемпературного максимума плотности ТСТД (450-550 К) имеет место значительное расхождение между амплитудами экспериментального и теоретического максимумов $[16,17]$, что объясняется неучтенными в теории токами проводимости $[3,4]$.

В связи с этим возникает необходимость в построении обобщенных аналитических выражений, описывающих процессы термостимулированной деполяризации в КВС с учетом неучтенных в $[1,16,17]$ нелинейных поляризационных эффектов, обусловленных взаимодействиями релаксационных мод плотности заряда, начиная с основной частоты переменного поля $[4,7]$, что и определяет актуальность выбранной темы исследования для усовершенствования методов диэлектрической спектроскопии. Прежде всего построение обобщенной нелинейной математической модели термостимулированной деполяризации актуально для областей аномально высоких электрофизических нелинейностей, проявляющихся в КВС в области сверхнизких температур (1-10 К) и слабых полей (100-1000 кВ/м) и в области сверхвысоких температур (550-1500 К) и сильных полей (10-1000 MB/м) [3, 4, 5, 7, 18].

Важным является вопрос об изучении процессов накопления и релаксации заряда в наноразмерных ППД (низкотемпературный электретный эфбект) при разработке топливных элементов водородной энергетики и для электрохимических технологий [19-25].

Математическое моделирование квантового переноса протонов в системах из тонких потенциальных барьеров с потенциальными ямами, содержащими квантово-размерные уровни энергии, важно при разработке схем резонансных туннельных диодов и квантовых полевых транзисторов на основе ППД, что актуально для оптоэлектроники и квантовой электроники [26-32].

Аномально высокие прозрачности потенциальных барьеров поперечного потенциального рельефа [18] на фоне достаточно высоких частот колебаний протонов в поперечных потенциальных ямах (порядка $\left.10^{13} \mathrm{c}^{-1}\right)[3,4,7,16,17]$ позволяют использовать эффекты наноразмерного состояния КВС [18] 
и некоторые другие квантовые размерные эффекты для создания сверхбыстродействующих цифровых или СВЧ-приборов с рабочими частотами более 1 ТГц [33-37].

Отсутствующие в [38-44] детализированные теоретические исследования свойств перспективных для техники высокотемпературных ионных суперпроводников (КЦК-керамика, YSZ, перовскиты и др.) могут быть выполнены методами нелинейной кинетической теории протонной релаксации [3-7].

Целью данной работы является усовершенствование методологии теоретических исследований термостимулированных токов деполяризации в КВС путем учета влияний нелинейностей высоких порядков теории возмущений (начиная со второго порядка по малому безразмерному параметру $[4,7])$ на кинетику процессов объемно-зарядовой поляризации в широком диапазоне полей $(100$ кВ/м - $1000 \mathrm{MB} / \mathrm{M})$ и температур (0-1500 К) [5-7].

Экспериментальное обоснование полученных аналитических выражений для плотности ТСТД, сопряженное с дополнительными процедурами численной оптимизации расчетных значений характеристических параметров релаксаторов [45], выходит за рамки данной работы и будет выполнено в дальнейшем.

Построение математической модели будем проводить в соответствии со схемой экспериментов по из- мерению температурных спектров токов термостимулированной деполяризации $[1,2]$. На первом этапе исследуем процесс поляризации диэлектрика под действием гармонически изменяющегося во времени однородного электрического поля $\mathrm{E}_{\text {pol }}(t)=\mathrm{E}_{0} \exp (\mathrm{i} \omega t)$ при блокирующих электродах. Температура поляризации принимается в эксперименте постоянной величиной $\mathrm{T}_{\text {pol }}$. На втором этапе исследуется процесс термостимулированной деполяризации в отсутствие внешнего электрического поля $\mathrm{E}(t)=0$ при нагреве кристалла по линейному закону $\mathrm{T}(t)=\mathrm{T}_{0}+c t$, где $c=$ const , $\mathrm{T}_{0}-$ начальная температура деполяризации, удовлетворяющая условию $\mathrm{T}_{0}<\mathrm{T}_{\text {pol }}$. Как правило, при измерении температурных спектров плотности ТСТД экспериментальные значения $\mathrm{T}_{0}$ принимаются вблизи азотной температуры (50-70 К), а температура поляризации устанавливается в пределах $\mathrm{T}_{\text {pol }} \approx 300-350 \mathrm{~K}[1,2]$. Скорость линейного нагрева кристалла $c \approx(0,1 \div 1) \frac{\mathrm{K}}{\mathrm{c}}[1,2]$.

2. Стационарная диэлектрическая проницаемость и поляризация диэлектрика

Принимаем поляризацию кристалла в бесконечном приближении по малому параметру теории возмущений $(\mathrm{k} \geq 1)$ на основной частоте $\omega$ переменного поля [4].

$$
P^{(\omega)}(t)=\frac{8 a q n_{0} \gamma}{\pi^{2}\left(1-\frac{8 n_{0} \phi \Lambda_{0} \gamma}{\pi^{2}}\right)} \times \sum_{\mathrm{n}=1}^{+\infty}\left[\frac{\sin ^{2}\left(\frac{\pi \mathrm{n}}{2}\right)}{\mathrm{n}^{2}\left(\frac{1}{\tau_{\mathrm{n}}}+\mathrm{i} \frac{\omega}{W^{(0)}}\right)}\right] \times \exp (\mathrm{i} \omega t) .
$$

В (1) комплексный безразмерный параметр $\Xi_{0}=\frac{8 n_{0} \phi \Lambda_{0} \gamma}{\pi^{2}}$ определяется рядом других фундаментальных параметров кинетической теории протонной релаксации [4]: $\phi=\frac{a \mathrm{q}}{\varepsilon_{0} \varepsilon_{\infty} \mathrm{E}_{0}}-$ величина, имеющая размерность, обратную равновесной концентрации релаксаторов (протонов) $n_{0} ; \gamma=\frac{\mu_{\mathrm{mob}}^{(1)} a \mathrm{E}_{0}}{\mathrm{D}_{\text {diff }}^{(0)}}=\varsigma_{0} \frac{W^{(1)}}{W^{(0)}}-$ малый безразмерный параметр теории возмущений; $\mathrm{D}_{\text {diff }}^{(0)}=a^{2} W^{(0)}, \mu_{\mathrm{mob}}^{(1)}=\frac{\mathrm{q} a^{2} W^{(1)}}{\mathrm{k}_{\mathrm{B}} \mathrm{T}}-$ коэффициенты диффузии и подвижности для протонов, вычисленные в «нулевом» приближении теории последовательных приближений [4]. Малый безразмерный параметр $\varsigma_{0}=\frac{\mathrm{qE}_{0} a}{\mathrm{k}_{\mathrm{B}} \mathrm{T}}$ имеет смысл параметра сравнения потенциальной (в электрическом поле) энергии и энергии теплового движения протонов; $\Lambda_{0}=\sum_{\mathrm{n}=1}^{\infty} \frac{\sin ^{2}\left(\frac{\pi \mathrm{n}}{2}\right)}{\mathrm{n}^{2}\left(\frac{1}{\tau_{\mathrm{n}}}+\mathrm{i} \frac{\omega}{W^{(0)}}\right)}-$ параметр взаимодействия релаксационных мо, на основной частоте $\omega[4,7]$. Безразмерное время релаксации $\tau_{\mathrm{n}}=\frac{\tau_{\mathrm{n}, \mathrm{D}} \tau_{\mathrm{M}}}{\tau_{\mathrm{n}, \mathrm{D}}+\tau_{\mathrm{M}}}$, соответствующее $\mathrm{n}$-й релаксационной моды объемной плотности заряда, раскрывается через диффузионное время релаксации $\mathrm{n}$-й моды 
$\tau_{\mathrm{n}, \mathrm{D}}=\frac{\tau_{\mathrm{D}}}{\mathrm{n}^{2}}$ и максвелловское время релаксации $\tau_{\mathrm{M}}=\frac{\varepsilon_{0} \varepsilon_{\infty} W^{(0)}}{\mathrm{q} n_{0} \mu_{\mathrm{mob}}^{(1)}}[4]$, где $\tau_{\mathrm{D}}=\left(\frac{\mathrm{d}}{\pi \mathrm{a}}\right)^{2}$ определяется с помощью толщины кристалла $\mathrm{d}$ и параметра решетки $a$; $\varepsilon_{\infty}-$ высокочастотная диэлектрическая проницаемость; q - заряд протона.
Расчет кинетических коэффициентов проводим в приближениях $l=0, l=1$, по малому параметру $\zeta(\mathrm{x} ; \mathrm{t})=\left|\frac{\mathrm{qE}(\mathrm{x} ; \mathrm{t}) a}{2 \mathrm{k}_{\mathrm{B}} \mathrm{T}}\right|<1$, с учетом как термически активируемых (классических), так и туннельных (квантовых) переходов протонов через параболический потенциальный барьер [6]:

$$
\begin{aligned}
& W^{(0)}(\mathrm{T})=\frac{\nu_{0}}{2}\left(\exp (-\mathrm{X})+\left\langle\mathrm{D}^{(0)}\right\rangle\right),\left\langle\mathrm{D}^{(0)}\right\rangle=\mathrm{X} \frac{\exp (-\Lambda)-\exp (-\mathrm{X})}{\mathrm{X}-\Lambda}, \\
& W^{(1)}(\mathrm{T})=\frac{\nu_{0}}{2}\left(\exp (-\mathrm{X})+\left\langle\mathrm{D}^{(1)}\right\rangle\right),\left\langle\mathrm{D}^{(1)}\right\rangle=\frac{\frac{\Lambda}{\mathrm{X}} \exp (-\Lambda)-\exp (-\mathrm{X})}{1-\frac{\Lambda}{\mathrm{X}}},
\end{aligned}
$$

где $\mathrm{X}=\frac{\mathrm{U}_{0}}{\mathrm{k}_{\mathrm{B}} \mathrm{T}}, \Lambda=\frac{\pi \delta_{0} \sqrt{\mathrm{mU}_{0}}}{\hbar \sqrt{2}}, \mathrm{~m}-$ масса протона; $\mathrm{U}_{0}$ - высота потенциального барьера (энергия активации протона на водородной связи); $\delta_{0}$ - ширина потенциального барьера; $v_{0}$ - линейная частота собственных колебаний протона в потенциальной яме $[5,6]$. Замечая, что $\tau_{\mathrm{M}}=\frac{1}{\theta}$, где $\theta=\phi \gamma n_{0}$, и принимая $\Xi_{0}=\frac{8 \Lambda_{0}}{\pi^{2} \tau_{\mathrm{M}}}$, а затем переходя к размерному времени релаксации $T_{\mathrm{M}}=\frac{\tau_{\mathrm{M}}}{W^{(0)}}$, в силу $\Xi=\frac{}{T W^{()}}$, а также с учетом равенства $T_{\mathrm{n}}=\frac{\tau_{\mathrm{n}}}{W^{(0)}}$ перепишем (1) в виде

$$
P^{(\omega)}(t)=\varepsilon_{0} \varepsilon_{\infty} E(t) \times \frac{\Gamma^{(\omega)}}{1-\Gamma^{(\omega)}} .
$$

В (2) введен комплексный параметр

$$
\begin{aligned}
& \Gamma^{(\omega)}=\frac{8}{\pi^{2} T_{M}} \sum_{n=1}^{\infty}\left[\frac{\sin ^{2}\left(\frac{\pi n}{2}\right)}{n^{2}\left(\frac{1}{T_{n}}+\mathrm{i} \omega\right)}\right] \text { с компонентами: } \\
& \Gamma_{1}^{(\omega)}(\mathrm{T})=\frac{4}{\pi^{2}} \sum_{\mathrm{n}=1}^{\infty}\left[\frac{\frac{T_{\mathrm{n}}}{T_{\mathrm{M}}}\left(1-(-1)^{\mathrm{n}}\right)}{\mathrm{n}^{2}\left(1+\omega^{2} T_{\mathrm{n}}^{2}\right)}\right], \\
& \Gamma_{2}^{(\omega)}(\mathrm{T})=\frac{4}{\pi^{2}} \sum_{\mathrm{n}=1}^{\infty}\left[\frac{\frac{\omega T_{\mathrm{n}}^{2}}{T_{\mathrm{M}}}\left(1-(-1)^{\mathrm{n}}\right)}{\mathrm{n}^{2}\left(1+\omega^{2} T_{\mathrm{n}}^{2}\right)}\right] .
\end{aligned}
$$

Представив поляризацию диэлектрика в общем виде [7]

$$
\mathrm{P}^{(\omega)}(\mathrm{t})=\varepsilon_{0}\left(\hat{\varepsilon}^{(\omega)}-\varepsilon_{\infty}\right) \cdot \mathrm{E}(\mathrm{t})
$$

в соответствии с (4), запишем комплексную диэлектрическую проницаемость

$$
\hat{\varepsilon}^{(\omega)}=\varepsilon_{\infty} \times \frac{1}{1-\Gamma^{(\omega)}} .
$$

Принимая $\omega=0$, в силу $\Gamma_{2}^{(\omega=0)}(\mathrm{T})=0$,

$\Gamma_{1}^{(\omega=0)}(\mathrm{T})=\frac{4}{\pi^{2}} \sum_{\mathrm{n}=1}^{\infty}\left[\frac{\frac{T_{\mathrm{n}}}{T_{\mathrm{M}}}\left(1-(-1)^{\mathrm{n}}\right)}{\mathrm{n}^{2}}\right]$ из (5), (6) получаем стационарные выражения: $\varepsilon_{\mathrm{S}}(\mathrm{T})=\varepsilon_{\infty} \mathrm{x}_{\mathrm{D}, \mathrm{M}} \cdot \operatorname{cth}\left(\mathrm{x}_{\mathrm{D}, \mathrm{M}}\right)$,

$$
\mathrm{P}_{\mathrm{S}}=\varepsilon_{0} \varepsilon_{\infty}\left(\mathrm{x}_{\mathrm{D}, \mathrm{M}} \cdot \operatorname{cth}\left(\mathrm{x}_{\mathrm{D}, \mathrm{M}}\right)-1\right) \cdot \mathrm{E}_{0},
$$

где $\mathrm{x}_{\mathrm{D}, \mathrm{M}}=\frac{\pi}{2} \sqrt{\eta(\mathrm{T})}$,

$$
\eta(\mathrm{T})=\frac{T_{\mathrm{D}}(\mathrm{T})}{T_{\mathrm{M}}(\mathrm{T})}=\frac{\mathrm{d}^{2} \mathrm{n}_{0} \mathrm{q}^{2}}{\pi^{2} \varepsilon_{0} \varepsilon_{\infty} \mathrm{k}_{\mathrm{B}} \mathrm{T}} \cdot \frac{W^{(1)}(\mathrm{T})}{W^{(0)}(\mathrm{T})} .
$$

\section{3. Термостимулированный ток деполяризации}

Исследования механизма термостимулированной деполяризации в КВС будем проводить на основании кинетического уравнения, описывающего процесс разрушения электретного заряда, накопленного во всем объеме диэлектрика в процессе поляризации (на первом этапе эксперимента) [6]

$$
\frac{\mathrm{d} P_{\text {depol }}(t)}{\mathrm{d} t}+2 \mathrm{~W}^{(0)}(t) \cdot P_{\text {depol }}(t)=0 .
$$


Начальная поляризация вычисляется согласно (7)

$$
P_{\text {depol }}(0)=\varepsilon_{0} \varepsilon_{\infty}\left(\mathrm{x}_{\mathrm{D}, \mathrm{M}}\left(\mathrm{T}_{p o l}\right) \cdot \operatorname{cth}\left(\mathrm{x}_{\mathrm{D}, \mathrm{M}}\left(\mathrm{T}_{p o l}\right)\right)-1\right) \cdot \mathrm{E}_{p o l} \cdot
$$

Решение уравнения (8) принимает вид

$$
P_{\text {depol }}(t)=P_{\text {depol }}(0) \cdot \exp \left(-2 \int_{0}^{\mathrm{t}} W^{(0)}(t)\right) \mathrm{d} t \text {. }
$$

Вычисление плотности тока термостимулированной деполяризации $J_{\text {depol }}(t)=-\frac{\partial P_{d e p o l}(t)}{\partial t}$ с учетом $c=\frac{\mathrm{dT}(t)}{\mathrm{d} t}$ окончательно дает

$$
J_{\text {depol }}(\mathrm{T})=2 P_{\text {depol }}(0) \cdot W^{(0)}(\mathrm{T}) \cdot \exp \left(-\frac{2}{\mathrm{c}} \int_{\mathrm{T}_{0}}^{\mathrm{T}} W^{(0)}(\mathrm{T})\right) \mathrm{dT} \text {. }
$$

Исследование интеграла $B(\mathrm{~T})=\int_{\mathrm{T}_{0}}^{\mathrm{T}} W^{(0)}(\mathrm{T}) \mathrm{dT}$ проводится численными методами.

Выражение (11) формально совпадает с известной формулой Буччи-Рива [2], однако научная новизна полученных результатов состоит в ряде принципиальных модельных отличий и дополнений. Во-первых, кинетические коэффициенты $W^{(0)}(\mathrm{T})$ вычисляются с учетом туннельных переходов релаксаторов (протонов) через параболический потенциальный барьер, и, во-вторых, начальная поляризация является достаточно сложной по структуре и информативной функции, отражающей влияния нелинейных поляризационных эффектов на кинетику процесса деполяризации. При этом выражение (11), в отличие от [2], работает в достаточно широком теоретическом диапазоне параметров полей (100 кВ/м - 1000 MB/м) и температур (0-1500 К) и позволяет выявить на теоретическом уровне поляризационные нелинейности, проявляющиеся в области сверхнизких температур (1-10 К) и сильных полей (100-1000 MB/м).

\section{Выводы}

1. Теоретически установлено, что статическая диэлектрическая проницаемость (СДП) материалов класса КВС в области сверхнизких (1-10 К) и сверхвысоких (550-1500 МВ/м) температур сложным образом (в отличие от линейной кинетической теории [1]) зависит от температуры поляризации.

2. В рамках феноменологической модели диэлектрической релаксации учтены влияния нелинейных поляризационных эффектов (взаимодействия релаксационных мод, квантовые переходы протонов и др.) на теоретическую плотность токов термостимулированной деполяризации в КВС.

3. Полученные теоретические результаты актуальны с точки зрения дальнейшего развития аналитических и компьютерных методов исследования и прогнозирования электрофизических свойств КВС как перспективных нелинейных материалов для ряда отраслей современной промышленности.

\section{Библиографический список}

1. Тонконогов М.П. Диэлектрическая спектроскопия кристаллов с водородными связями. Протонная релаксация // УФН. 1998. Т. 168, № 1.

2. Калытка В.А., Коровкин М.В. Протонная проводимость : монография. Germany, 2015 [Электронный ресурс]. URL : http://www.lap-publishing.com.

3. Калытка В.А. Математическое описание нелинейной релаксационной поляризации в диэлектриках с водородными связями // Вестник Самарского ун-та. Естественнонаучная серия. 2017. Т. 23. № 3.

4. Калытка В.А. Нелинейные кинетические явления при поляризации твердых диэлектриков // Вестник Московского гос. обл. ун-та (МГОУ). Серия : Физика-математика. 2018. № 2 .

5. Калытка В.А. Аналитическое исследование нелинейных электрофизических процессов в протонных полупроводниках и диэлектриках // Известия Алт. гос. ун-та. Физика. 2019. № 1 (105).
6. Калытка В.А., Коровкин М.В. Дисперсионные соотношения для протонной релаксации в твердых диэлектриках // Известия вузов. Физика. 2016. Т. 59. № 12.

7. Калытка В.А., Коровкин М.В., Мехтиев А.Д., Алькина А.Д. Детальный анализ нелинейных диэлектрических потерь в протонных полупроводниках и диэлектриках // Вестник Моск. гос. обл. ун-та (МГОУ). Серия : Физика-математика. 2017. № 4.

8. Белоненко М.Б. Особенности нелинейной динамики лазерного импульса в фоторефрактивном сегнетоэлектрике с водородными связями // Квантовая электроника. 1998. Т. 25. № 3.

9. Левин А.А., Долин С.П., Зайцев А.Р. Распределение заряда, поляризация и свойства сегнетоэлектриков типа $\mathrm{KH}_{2} \mathrm{PO}_{4}$ (KDP) // Химическая физика. 1996. Т. 15.

10. Лебедев Н.Г., Литинский А.О. Модель ионно-встроенного стехиометрического кластера для расчета электронного строения ионных кристаллов // Физика твердого тела. 1996. Т. 38. № 3. 
11. Лебедев Н.Г., Белоненко М.Б. Строение и электронная структура сегнетоэлектриков KDP-типа // Вестник Волгоградского гос. ун-та. Серия : Математика-физика. 1997. № 2 .

12. Прасолов Б.Н., Сафонова И.А. Влияние скорости и направления прохождения фазового перехода второго рода на диэлектрические потери в кристаллах ТГС // Известия АН СССР. Серия : Физика. 1993. Т. 57.

13. Трюхан Т.А., Стукова Е.В., Барышников С.В. Диэлектрические свойства триглицинсульфата в пористых матрицах // Известия Самарского научн. центра РАН. Серия : Физика и электроника. 2010. Т. 12. № 4.

14. Стеханова Ж.Д., Яценко О.Б., Миловидова С.Д., Сидоркин А.С., Рогазинская О.В. Свойства кристаллов триглицинсульфата, выращенных из водных растворов // Журнал прикладной химии. 2005. Т. 78. № 1.

15. Яценко О.Б., Чудотворцев И.Г., Стеханова Ж.Д. Влияние температуры и содержания воды на свойства кристаллов триглицинсульфата // IV Междунар. симпозиум «Фракталы и прикладная синергетика». М., 2005.

16. Тонконогов М.П., Исмаилов Ж.Т., Тимохин В.М., Фазылов К.К., Калытка В.А., Баймуханов 3.К. Нелинейная теория спектров термостимулированных токов в сложных кристаллах с водородными связями // Известия вузов. Физика. 2002. № 10.

17. Тонконогов М.П., Кукетаев Т.А., Фазылов К.К., Калытка В.А. Квантовые эффекты при термодеполяризации в сложных кристаллах с водородными связями // Известия вузов. Физика. 2004. № 6.

18. Анненков Ю.М., Калытка В.А., Коровкин М.В. Квантовые эффекты при миграционной поляризации в нанометровых слоях протонных полупроводников и диэлектриков при сверхнизких температурах // Известия вузов. Физика. 2015. Т. 58. № 1.

19. Пальгуев С.Ф. Высокотемпературные протонные твердые электролиты. Екатеринбург, 1998.

20. Timofeeva N.I., Demin A.K. Modelling of SOFC Operation in Variable Regime // In 1-st European Solid Oxide Fuel Cell Forum / U. Bossel, Editor, Druckerei J. Kinzel, Gottingen, Germany, 1994.

21. Коровин Н.В. Электрохимическая энергетика. М., 1991.

22. Demin A.K. In Hydrogen Energy Progress IX. T.N. Veziroglu, C. Derive and J. Pottier, Editors. MCI, Paris, 1992.

23. Зюбина Т.С., Шилов Г.В., Добровольский Ю.А., Леонова Л.С., Мебель А.М. Моделирование протонного транспорта в ортоиодной и ортотеллуровой кислотах и их солях // Электрохимия. 2003. Т. 39. № 4.

24. Ярославцев А.Б. Основные направления разработки и исследования твердых электролитов // Успехи химии. 2016. Т. 85. № 11.

25. Ярославцев А. Б. Протонная проводимость неорганических гидратов // Успехи химии. 1994. Т. 5. № 63.
26. Абрикосов А.А. Резонансное туннелирование в высокотемпературных сверхпроводниках // Успехи физических наук. 1998. Т. 168. № 6.

27. Силин А.П. Полупроводниковые сверхрешетки // Успехи физических наук. 1985. Т. 147. № 3.

28. Усанов Д.А., Скрипаль А.В. Физические основы наноэлектроники : уч. пос. для студентов факультета нанои биомедицинских технологий. Саратов, 2013.

29. Brown E.R., Söderström J.R., Parker C.D., Mahoney L.J., Molvar K.M., McGill T.C. Oscillations up to $712 \mathrm{GHz}$ in In-As/AlSb resonant-tunneling diodes// J. Applied Physics Letters, 1991. V. 58.

30. Попов В.Г. Токовые неустойчивости в резонанснотуннельных диодах // Успехи современной радиоэлектроники. 2010. № 6.

31. Physics of Quantum Electron Devices / ed. by F. Capasso. Berlin, 1990.

32. Brown E. R. Resonant tunneling in high-speed doublebarrier diodes // Hot Electrons in Semiconductor Heterostructures / ed. by J. Shah. Boston, 1991.

33. Ozbay E., Bloom D. M., Diamond S. K. Resonant tunneling in Semiconductors: Physics and Applications. New York, 1991.

34. Тагер А.С. Размерные квантовые эффекты в субмикронных полупроводниковых структурах и перспектива их применения в электронике СВЧ. Ч. І. Физические основы // Электронная техника. Сер. 1 : Электроника СВЧ. 1987. № 9 (403).

35. Тагер А.С. Размерные квантовые эффекты в субмикронных полупроводниковых структурах и перспектива их применения в электронике СВЧ. Ч. ІІ. Резонанснотуннельные диоды и транзисторы // Электронная техника. Сер. 1 : Электроника СВЧ. 1988. № 2 (406).

36. Иогансен Л.В. О возможности резонансного прохождения электронов в кристаллах через систему барьеров // ЖЭТФ. 1963. Т. 45.

37. Иогансен Л.В. О резонансном туннелировании электронов в кристаллах // ЖЭТФ. 1964. Т. 47. № 1.

38. Анненков Ю.М., Ивашутенко А.С., Власов И.В., КабышевА.В. Электрические свойства корундо-циркониевой керамики // Известия Том. политех. ун-та. 2005. Т. 308. № 7.

39. Кытин В.Г., Кульбачинский В.А., Кондратьева Д.Ю., Павликов А.В., Григорьев А.Н., Манкевич А.С., Корсаков И.Е. Прыжковый перенос дырок в керамических образцах $\mathrm{CuCrO} 2$, легированного магнием // Ученые записки физ. фак. Мос. ун-та. 2018. № 1.

40. Пальгуев С.Ф. Высокотемпературные протонные твердые электролиты. Екатеринбург, 1998.

41. Khromushin I.V., Aksenova T.I., Baykov Yu.M. Regularities of oxygen and water thermal desorption from barium cerate doped by neodymium, samarium, and gadolinium//Russian Journal of Electrochemistry. 2017. Vol. 53, No. 6 . 
42. Хромушин И.В., Аксенова Т.И. Влияние низкоэнергетических ионов аргона на проводящие свойства YSZ // Вестник НЯЦ РК. 2017. Вып. 1.

43. Хромушин И.В., Аксенова Т.И. Влияние облучения ионами кислорода и аргона на проводящие свойства диоксида циркония, стабилизированного иттрием (YSZ) // Междунар. научн. форум «Ядерная наука и технологии». Алматы. 2017.
44. Ziegler J.F., Biersack J.P., Ziegler M.D., SRIM The Stopping and Range of Ions in Matter. 2012.

45. Калытка В.А. Разработка схемы численного расчета параметров нелинейных электрофизических процессов методом минимизации функции сравнения // Пространство, время и фундаментальные взаимодействия. М., 2018. № 3 (24). 